\title{
Stress-Induced Eating Dampens Physiological and Behavioral Stress Responses
}

\author{
Laura E. Finch and A. Janet Tomiyama
}

Department of Psychology, University of California, Los Angeles, CA, USA

\section{INTRODUCTION}

Perceived stress influences health behaviors across multiple domains, with high stress levels associated with a high-fat diet, less frequent exercise, and cigarette smoking [1]. A recent national survey found that $44 \%$ of adults report feeling greater stress now than they did 5 years ago, and $39 \%$ report eating too much or eating unhealthy foods due to feelings of stress in the past month [2]. Chronic life stress is associated with increased consumption of energy- and nutrient-dense "comfort foods," particularly high-fat and high-sugar foods [3]. This altered eating behavior in times of stress is referred to as stress-induced eating, emotional eating, or comfort eating. While the first term refers to eating behavior being specifically altered in response to feelings of stress, the latter two terms may be used to describe altered eating in response to a range of emotional states (e.g. stress, sadness, anger, or boredom). Here we primarily focus on stress-induced eating because it is clearly operationally defined across species and is most commonly examined in existing research.

Acute psychological stress in humans is associated with increased food intake even in the absence of hunger [4]. For example, in student populations, exams or high workload have been associated with higher energy and fat intake [5] or a decreased quality of dietary nutrition [6]. Furthermore, chronic stress exposure also promotes stress-induced eating behavior. Psychological stress due to interpersonal and workrelated daily hassles is associated with an increased consumption of high-fat and high-sugar between-meal snacks and with decreased consumption of main meals and vegetables [7].
Not all individuals increase their food intake in response to stress; in studies of self-reported stress and food intake, approximately $40-70 \%$ of humans report increasing and 30-60\% report decreasing their food intake under stress $[8,9]$. Researchers have yet to fully identify which factors account for these individual differences, but body mass index (BMI) may matter. Data from the Whitehall II study indicated that under stress conditions, individuals with higher initial BMI tend to gain weight and vice versa [10]. Twin studies indicate that stress-induced eating may also be partially heritable [11]. Some researchers have also observed gender [12] and racial/ethnic differences [13] in the levels of stressinduced eating, with females and black individuals engaging in more emotional eating, and others not [14]. Individuals characterized by restrained eating and sensitivity to chronic stress may also be particularly susceptible to stress-induced eating [4]. Regardless of whether the overall amount of food consumption changes under stress, all individuals (including nonhuman animals) alter their eating habits to consume palatable foods characterized by high-calorie, high-sugar, and high-fat content [15].

Persistent stress-induced eating behavior over time results in intra-abdominal obesity [16], which is strongly associated with metabolic syndrome, hypertension, type 2 diabetes mellitus, cardiovascular disease, morbidity, and mortality $[17,18]$. The association of stress-induced eating with increased visceral fat accumulation has motivated the development of stress-induced eating interventions on the premise that stress eating is unequivocally harmful [19]. However, there is a growing literature demonstrating the potentially salutary role of stressinduced eating behavior in dampening both physiological and behavioral stress responses. 
In this chapter, we review cross-species literatures describing how experiences of stress affect eating behavior and fat distribution, and vice versa. First, we review research demonstrating the effects of both acute and chronic stressors in promoting stress-induced eating. Next, we discuss how repeated engagement in stressinduced eating under chronic stress conditions leads to the accumulation of visceral fat, as evidenced primarily in studies of nonhuman animals. We then review the burgeoning literature supporting the joint role of stressinduced eating and abdominal fat in attenuating stress responses, focusing on glucocorticoids and insulin as endocrine promoters of these processes. We go on to describe potential affective and neural mechanisms for perpetuating the cycle of stress-induced eating. The vast majority of data regarding the physiological and behavioral effects of stress-induced eating comes from studies of nonhuman animals. However, to the extent that these effects have been tested in humans, consistent results have been observed. We conclude our discussion with a summary of the remaining gaps in the literature and suggestions for future research.

\section{GLUCOCORTICOIDS AND INSULIN PROMOTE STRESS-INDUCED EATING}

Both physical and psychological stressors are known to stimulate the hypothalamic-pituitary-adrenal (HPA) axis. Stress-induced activation of the HPA axis begins with the release of corticoliberin [corticotropin-releasing factor (CRH), encoded by the CRH gene] from the hypothalamus, which stimulates the release of adrenocorticotropic hormone (ACTH) from the anterior pituitary gland. ACTH then circulates through the bloodstream to the adrenal cortex, where it stimulates glucocorticoid secretion [20].

Increased glucocorticoid secretion following HPA axis activation appears to be a central pathway through which stress promotes the consumption of palatable foods. In a sample of healthy premenopausal women, individuals who exhibited a higher total cortisol output in response to a laboratory stressor consumed more calories that day compared to those with a lower total cortisol response [21]. Furthermore, oral administration of glucocorticoids in healthy white males has been shown to increase their intake of total energy, carbohydrate, and protein, but not of fat [22]. Dose-response effects of glucocorticoid concentrations on eating behavior may be robustly tested through the study of adrenalectomized organisms, as they lack the capability to naturally produce glucocorticoids via the adrenal glands. In adrenalectomized rats, the administration of corticosterone directly affects the consumption of both saccharin and sucrose in a dose-response manner, with larger corticosterone doses resulting in a greater proportional intake of these substances $[23,24]$.

Importantly, increased glucocorticoid concentrations stimulate insulin secretion [25]. In a series of experiments in rats, La Fleur and colleagues identified complex interactions between corticosterone and insulin in the regulation of food intake, finding that glucocorticoids elicit doserelated increases in total caloric intake, and that increased insulin concentrations stimulated by glucocorticoids specifically regulate the amount of fat consumed [26]. These effects are evidenced in studies of adrenalectomized, diabetic rats, in which concurrent glucocorticoids and insulin promote a shift in intake toward fat and sugar, whereas glucocorticoids in the absence of insulin promote the consumption of bland rat chow. Therefore, it seems that glucocorticoids function to increase general food-associated drives, while insulin influences preferences for which types of foods are consumed.

\section{GLUCOCORTICOIDS AND INSULIN PROMOTE VISCERAL FAT ACCUMULATION}

Insulin and glucocorticoids also have complex interactions in fat accumulation. These hormones have acutely antagonistic effects, with glucocorticoids inhibiting energy storage and insulin promoting energy storage [27]. However, in the location of fat stores, insulin and high glucocorticoids act synergistically to promote visceral fat accumulation, especially under chronically stressful conditions $[28,29]$. Cortisol binds to glucocorticoid receptors and activates lipoprotein lipase (LPL), thus increasing triglyceride accumulation in adipocytes. In the presence of insulin, cortisol also inhibits the lipidmobilizing system, resulting in triglyceride retention [28]. Concurrent cortisol and insulin also promote visceral fat accumulation indirectly by inhibiting the activity of somatotropin/growth hormone, which otherwise exerts lipolytic effects [30]. These processes are amplified in intra-abdominal adipose tissue, where there is a particularly high density of glucocorticoid receptors compared to other fat depots [28]. Visceral fat accumulation further perpetuates this cycle by providing increased intracellular glucocorticoids [31]. These effects are particularly apparent in patients with Cushing syndrome, who are both hypercortisolemic and hyperinsulinemic and have high levels of intra-abdominal obesity [32].

These processes have been demonstrated experimentally. In rats, the administration of high glucocorticoid concentrations in the presence of insulin increases fat stores [26,33]. Although no experimental studies are known to have been conducted in humans, preliminary evidence suggests that some of the same processes are at work. For example, the synergistic 
role of these hormones in promoting stress-induced eating and weight gain is supported in a sample of healthy medical students who categorized themselves as consistently eating either more or less during stress [34]. Students that characterized themselves as normatively eating more during stress tended to gain more weight and also demonstrated increases in urinary cortisol and insulin levels during exam periods, controlling for baseline. Although this study did not test for expected increases in fat and sugar consumption related to increased insulin, the increase in total food intake self-reported by the "eat-more" group is consistent with their corresponding elevated cortisol concentrations.

\section{STRESS-INDUCED EATING DAMPENS PHYSIOLOGICAL AND BEHAVIORAL STRESS RESPONSES}

There is increasing evidence from animal models supporting an important therapeutic role of comfort eating in inhibiting the HPA axis response to both acute and chronic stressors. Studies in nonhuman animals have demonstrated what Dallman and colleagues have termed a chronic stress response network model, that is, a process through which stress-induced eating decreases neuroendocrine activity associated with the physiological stress response [33]. For example, in rats exposed to chronic restraint stress, the provision of palatable lard or sucrose has been shown to dampen stress responses compared to a diet of less palatable chow [35]. In particular, there is an attenuated physiological stress response in the form of reduced ACTH secretion. The consumption of palatable food may reduce HPA outflows even in the absence of stress; nonstressed rats given the palatable diet showed reduced $\mathrm{Crh}$ expression compared to nonstressed rats given chow. Similarly, in rats exposed to chronic unpredictable physical stress, the provision of daily limited access to sucrose or saccharin solutions decreased $\mathrm{Crh}$ mRNA expression in the paraventricular nucleus of the hypothalamus [36]. Furthermore, sucrose ingestion reduced the plasma corticosterone response to restraint in these chronically stressed rats. Additional research suggests that a palatable diet is also effective in dampening physiological responses to acute stressors. A study in rats tested the effects of prior access (7 days) to chow versus palatable foods on HPA axis activation following $4 \mathrm{~h}$ of acute restraint stress [37]. Rats with prior access to palatable foods showed inhibited ACTH and corticosterone responses, as well as reduced $\mathrm{Crh}$ mRNA in the hypothalamus and oval nucleus of the bed nuclei of the stria terminalis. In summary, these studies suggest that the consumption of palatable foods decreases endocrine responses to both acute and chronic physical stressors.
In addition to attenuating physiological stress responses, the consumption of palatable food has also been shown to ameliorate behavioral responses to stressors. In rats, the ingestion of palatable food reduces the effects of chronic maternal separation stress, decreasing anxiety and depressive-like behaviors [38]. Likewise, in mice exposed to chronic unpredictable social defeat and overcrowding stress, concurrent subjection to a high-fat diet decreased the expression of anxiety and depressivelike behaviors, compared to mice on a concurrent low-fat diet. However, a high-fat diet appears to protect from some but not other behavioral effects of chronic social stress; for example, social avoidance and anhedonic behavior were not affected [39]. Finally, in male rats, the short-term (7 days) intake of a high-fat diet reduced acute behavioral anxiety response to an elevated plus maze stressor. This effect appears to be highly dependent upon macronutrient content, as comparison highcarbohydrate and high-protein diets did not decrease anxiety behaviors [40]. These results consistently support the protective role of a palatable diet in reducing anxiety and depressive behaviors in response to both acute and chronic psychosocial stressors. While no known studies to date have experimentally tested these behavioral effects in humans, these findings in rodent models may be particularly relevant to human populations; compared to the administration of physical stressors, the psychosocial stress paradigms employed in these behavioral studies bear a closer resemblance to the types of stressors naturally experienced by some humans.

\section{REINFORCEMENT OF STRESS-INDUCED EATING VIA AFFECTIVE RESPONSES, THE REWARD SYSTEM, AND ENHANCED MEMORY}

Stress and food intake interact in a bidirectional manner. Just as stress and mood can alter eating behavior, food choice can conversely impact stress and mood, whether deliberate or unintended. As reviewed by Gibson [41], choosing to eat particular foods can alter mood via sensory or hedonic effects, associated social context, cognitive expectations, psychological distraction, changes in appetite, or nutritional modulation of brain function. For example, high-sugar, high-fat foods low in protein may ameliorate stress via enhanced functioning of the serotonergic system. Laboratory experiments have also demonstrated that sweet-tasting substances may provide analgesic effects during acute stressors, e.g. increasing pain threshold latency and pain tolerance during cold-pressor tasks [42,43]. Taken together, these findings suggest a general capability of comfort foods to elicit desirable affective or sensory responses in humans, which may function to reinforce stress-induced eating behavior. 
The experience of eating can be both pleasurable and rewarding. Indeed, the addiction literature suggests that repeated activation of neural reward circuitry may also drive stress-induced eating. Food intake stimulates the release of endogenous opiates and activates neural substrates such as dopamine in a similar manner to drugs of abuse, albeit with differences in intensity [44]. Consequently, opiate release may serve to protect an organism from the detrimental effects of stress by decreasing HPA axis activity and dampening the stress response. In a reward-based stress eating model, repeated stimulation of reward pathways coinciding with stress-induced eating may result in neurobiological adaptations that promote the compulsive nature of overeating [31]. Reward-related consumption can result in caloric intake exceeding requirements, and may be a critical etiological pathway in the development of obesity, in particular abdominal obesity [45].

In addition, the cycle of stress-induced eating may also be perpetuated by learning and memory via the action of glucocorticoids. Considerable evidence has accumulated indicating that glucocorticoids are crucially involved in the regulation of memory, specifically enhancing memory consolidation of emotionally arousing experiences [46]. Thus, when stress promotes glucocorticoid-induced palatable food intake, glucocorticoids also facilitate the formation of cognitive associations between comfort food indulgence and subsequent positive affect.

\section{ROLE OF GLUCOCORTICOIDS AND VISCERAL FAT IN DAMPENING STRESS RESPONSES}

In addition to their role in promoting stress-induced eating and memory formation, glucocorticoids are also crucial in the development of visceral fat accumulation [28], which in turn attenuates stress responses [33]. The effect of stress-induced glucocorticoid secretion on subsequent ACTH secretion is dependent upon the chronicity of stressors. Within hours of acute stressors, glucocorticoids directly inhibit further activity in the HPA axis [47], but across the course of days the chronic actions of glucocorticoids on the brain are directly excitatory [33]. As summarized by Dallman and colleagues [33], chronically high glucocorticoid concentrations act in three functionally congruent ways: (1) increasing the expression of $\mathrm{Crh}$ mRNA in the amygdala; (2) increasing the salience of pleasurable or compulsive activities, including the motivation to consume "comfort food"; and (3) systematically increasing visceral fat stores. These visceral stores then send a negative feedback signal to inhibit both catecholamines in the brainstem and $\mathrm{CRH}$ expression in hypothalamic neurons that regulate
ACTH. Indeed, studies in chronically stressed rats have consistently demonstrated a strong negative correlation between intra-abdominal fat stores and hypothalamic CRH expression [16,48]. Therefore, it seems that under chronic stress conditions, abdominal fat serves as a surrogate for the negative feedback signal of glucocorticoids that is normally present under acute stress conditions, thus inhibiting further HPA activation. Although the particular signal to the brain that represents increased abdominal fat stores remains unidentified, it is apparent that this signal does indeed act in the brain to decrease the adverse effects of the chronic stress response, plausibly promoting feelings of wellbeing [16].

While stress-induced palatable eating and greater abdominal fat stores have consistently been shown to reduce signs of stress in rodent models, there is a dearth of research examining these processes in other organisms. However, preliminary evidence suggests that these processes are indeed conserved across species.

In a study by our group [50], women with high chronic perceived stress reported more emotional eating and had significantly greater BMI and sagittal diameter in comparison to low-stress women. Emotional eating behavior was measured using the Dutch Eating Behavior Questionnaire (DEBQ) [51], a well-validated measure of eating behavior. In addition to these cross-sectional results, a laboratory component of the study also measured HPA axis activation in response to an acute laboratory stressor, the Trier Social Stress Test (TSST) [52]. This is a procedure designed to induce psychological and physiological stress responses via the performance of both a speech task and mental arithmetic task in front of an evaluative audience. Following the TSST, the chronic high-stress women showed a blunted cortisol response and lower diurnal cortisol levels compared to low-stress women. While these findings are consistent with rodent models, this study is limited by its lack of direct measurement of eating behavior following stressor exposure.

Two additional studies in humans expand upon previous findings by directly measuring food intake after exposure to an acute laboratory stressor. The first study compared female students with extremely high or low scores on emotional eating, as measured by the DEBQ [53]. The study exposed participants to a modified TSST, subsequently assessing total cortisol response to the stressor, as well as their food intake. Results revealed emotional eating to be a significant moderator of the relationship between cortisol stress reactivity and food intake. High emotional eaters with a blunted cortisol response consumed more food after stress than those with an elevated cortisol response, whereas no relationship was found in low emotional eaters. Thus, these results are consistent with previous studies 
demonstrating a relationship between blunted cortisol stress response and increased stress-induced eating in high emotional eaters.

Using a similar experimental design, Tryon et al. assessed both food intake and total cortisol response following administration of the TSST [54]. In this sample of healthy, perimenopausal women, responses to the stressor were compared on the basis of high vs. low selfreported chronic stress levels, as measured by the Wheaton Social Stress Index [55]. Women with high chronic stress and low cortisol reactivity consumed more calories from chocolate cake in response to the stressor. Moreover, the combination of high chronic stress and low cortisol reactivity was positively associated with total fat mass and regional fat percentage. This finding supports the previously discussed concept of a metabolic feedback pathway, in which a signal sent from adipose tissue inhibits activation of the HPA axis. In conclusion, early studies in humans repeatedly show correlations between high chronic stress, high emotional eating, greater abdominal fat, and decreased cortisol responses to acute laboratory stressors. Future studies should expand upon this work by investigating how stress-induced eating and abdominal fat stores affect responses to naturalistic stressors in humans.

Additional evidence from an epidemiological study supports the chronic stress response network model. In black individuals, the relationship between stressors and meeting major-depression criteria was buffered in obese individuals, compared to nonobese individuals [13]. Although this study was limited by its lack of a direct measurement of eating behavior, it suggests a significant paradox in which obesity (a proxy for overeating) serves a protective role for mental health outcomes, while simultaneously contributing to negative physical health outcomes in the form of higher rates of chronic conditions, morbidity, and mortality. Further research is needed to test the potential protective role of obesity and stress-induced eating behavior for mental health by specifically exploring its capacity for dampening psychological stress responses.

While stress-induced eating has been shown to reliably attenuate stress responses in rodents, and preliminarily in humans, these effects may be variable in other species. For example, in a study of 10 adult female rhesus monkeys, stress-induced eating was shown to decrease behavioral, but not physiological stress responses [56]. For a period of 3 weeks of social subordination stress exposure, the females were assigned to either a low-calorie diet or to a choice condition with the provision of both a low- and high-calorie diet. Food intake, cortisol secretion, and socioemotional behavior were assessed over time in both subordinate females and nonstressed dominant females. Of the females in the diet-choice condition, subordinate females consumed more calories from the high-calorie diet and gained more weight compared to dominant females. However, the provision of a high-calorie diet was associated with a flattening of the diurnal cortisol rhythm and did not decrease the cortisol response to acute social separation. Although these physiological findings are inconsistent with previous work in other species, diet condition did indeed influence behavioral stress responses over time. While females in the low-calorie diet condition exhibited increasing rates of anxiety-like behaviors, females in the diet-choice condition showed decreasing rates of these behaviors. Interestingly, rates of aggressive behavior directed at other females were significantly higher for females in the no-choice condition with only the lowcalorie diet available. In summary, stress-induced eating may decrease physiological stress responses in some, but not all, species. Nevertheless, cross-species data consistently show decreased behavioral stress responses with the provision of a palatable diet.

\section{CONCLUSION AND FUTURE DIRECTIONS}

There is strong evidence across species that both acute and chronic stressors promote increased consumption of palatable comfort foods. Furthermore, studies in rodents have consistently shown that chronic persistence in stress-induced eating behavior results in increased visceral fat accumulation, which functions to attenuate HPA axis activation in response to stressors. These effects have only just begun to be tested in humans; however, preliminary evidence is consistent with the dampened stress responses demonstrated in rodents. Of the few studies that have investigated these processes in humans, the outcome measures of stress have been responses to acute social evaluative stressors administered in the laboratory setting. Additional research is needed to explore how stress-induced eating might reduce experiences of stress related to more heterogeneous and naturally occurring stressors outside the laboratory.

In addition to exploring how the chronic stress response network functions in humans, future studies should also include experimental designs aimed to investigate multiple stress systems. Previous research has primarily focused on HPA axis activation as the principal outcome measure of dampened stress responsivity. However, autonomic and immune system activity is also heavily influenced by exposure to stressors. Therefore, future work should evaluate the potential role of stressinduced eating in similarly attenuating responses to stress across these additional physiological systems.

Furthermore, little is known about how stress dampening may be particularly dependent upon the macronutrient content of palatable foods. Are characteristically 
high-sugar, high-fat foods necessary for decreasing stress responsivity or can high-protein, or simply highcalorie, foods also elicit these responses?

Future research should also carefully delineate which specific types of circumstances or emotional exposures are most responsible for triggering the stress-induced eating process. For example, is general stress driving this behavior, or do certain characteristic types of stress, such as uncontrollable or unpredictable stress experiences, have the same effect? Alternatively, are experiences with other types of negative emotion (e.g. anger, sadness, or worry), or even positive emotion, also effective in eliciting stress-induced eating and its corresponding attenuated stress response?

Similarly, researchers should investigate which individuals are particularly (1) susceptible to stress-induced eating and (2) effective in dampening stress responses via stress-induced eating. Some evidence supports genetics, BMI, and demographic characteristics such as gender and race/ethnicity as potential candidates for the former; virtually no research exists on the latter.

Finally, existing research has yet to conclusively identify the peripheral negative feedback signal to the brain that promotes the inhibition of further HPA axis activation during chronic stress exposure [for a review, see 56]. Potential signal mechanisms and structures include insulin activity, vagus nerve stimulation, and neural alterations in the activity of the prefrontal cortex.

Although obesity is known to develop from myriad genetic and environmental factors, the growing literature on stress-induced eating will elucidate the complex relationships between stress, eating, and obesity. If stress-induced eating leads to the consumption of food in excess of nutritive requirements, then this behavior may culminate in abdominal weight gain and obesity. However, the dampened physiological and behavioral stress responses demonstrated in numerous studies suggest that stress-induced eating provides short-term amelioration of stress. This ostensible benefit of stress-induced eating has important implications for evaluating whether attempts at stress-induced eating intervention are an appropriate response to the behavior. Further research evaluating the causes and effects of stress-induced eating will improve our understanding of why individuals engage in this behavior and how it functions as a contributing factor in the etiology of obesity.

\section{References}

[1] Ng DM, Jeffery RW. Relationships between perceived stress and health behaviors in a sample of working adults. Health Psychol 2003;22:638-42.

[2] American Psychological Association Press Release. Stress in America: Our health at risk. <http:/ / www.apa.org/news/press / releases/stress/2011/final-2011.pdf> [accessed 02.04.13].
[3] Torres SJ, Nowson CA. Relationship between stress, eating behavior, and obesity. Nutrition 2007;23:887-94.

[4] Rutters F, Nieuwenhuizen AG, Lemmens SG, Born JM, WesterterpPlantenga MS. Acute stress-related changes in eating in the absence of hunger. Obesity 2008;17:72-7.

[5] Michaud C, Kahn JP, Musse N, Burlet C, Nicolas JP, Mejean L. Relationships between a critical life event and eating behaviour in high-school students. Stress Med 1990;6:57-64.

[6] Weidner G, Kohlmann CW, Dotzauer E, Burns LR. The effects of academic stress on health behaviors in young adults. Anxiety Stress Copin 1996;9:123-33.

[7] O'Connor DB, Jones F, Conner M, McMillan B, Ferguson E. Effects of daily hassles and eating style on eating behavior. Health Psychol 2008;27:S20-31.

[8] Oliver G, Wardle J. Perceived effects of stress on food choice. Physiol Behav 1999;66:511-5.

[9] Kandiah J, Yake M, Willett H. Effects of stress on eating practices among adults. Fam Consum Sci Res J 2008;37:27-38.

[10] Kivimaki M, Head J, Ferrie JE, et al. Work stress, weight gain and weight loss: evidence for bidirectional effects of job strain on body mass index in the Whitehall II study. Int J Obes 2006;30:982-7.

[11] Sung J, Lee K, Song YM, Lee MK, Lee DH. Heritability of eating behavior assessed using the DEBQ (Dutch Eating Behavior Questionnaire) and weight-related traits: the healthy twin study. Obesity 2010;18:1000-5.

[12] Snoek HM, Van Strien T, Janssens JM, Engels RC. Emotional, external, restrained eating and overweight in Dutch adolescents. Scand J Psychol 2007;48:23-32.

[13] Jackson JS, Knight KM, Rafferty JA. Race and unhealthy behaviors: chronic stress, the HPA axis, and physical and mental health disparities over the life course. Am J Public Health 2010;100:933-9.

[14] Ledoux T, Watson K, Baranowski J, Tepper BJ, Baranowski T. Overeating styles and adiposity among multiethnic youth. Appetite 2011;56:71-7.

[15] Dallman MF. Stress-induced obesity and the emotional nervous system. Trends Endocrinol Metab 2010;21:159-65.

[16] Dallman MF, Pecoraro NC, la Fleur SE. Chronic stress and comfort foods: self-medication and abdominal obesity. Brain Behav Immun 2005;19:275-80.

[17] Wajchenberg BL. Subcutaneous and visceral adipose tissue: their relation to the metabolic syndrome. Endocr Rev 2000;21:697-738.

[18] Björntorp P. " Portal" adipose tissue as a generator of risk factors for cardiovascular disease and diabetes. Arteriosclerosis 1990;10:493-6.

[19] Daubenmier J, Kristeller J, Hecht FM, Maninger N, Kuwata M, Jhaveri $\mathrm{K}$, et al. Mindfulness intervention for stress eating to reduce cortisol and abdominal fat among overweight and obese women: an exploratory randomized controlled study. J Obes 2011;2011:651936. http://dx.doi.org/10.1155/2011/651936.

[20] Lovallo WR, Thomas TL. Stress hormones in psychophysiological research: emotional, behavioral, and cognitive implications. In: Cacioppo JT, , Tassinary LG, Berntson GG, editors. Handbook of psychophysiology. 2nd ed.. New York: Cambridge University Press; 2000. p. 342-67.

[21] Epel E, Lapidus R, McEwen B, Brownell K. Stress may add bite to appetite in women: a laboratory study of stress-induced cortisol and eating behavior. Psychoneuroendocrinology 2001;26:37-49.

[22] Tataranni P, Larson D. Effects of glucocorticoids on energy metabolism and food intake in humans. Am J Physiol 1996;271: E317-25.

[23] Bhatnagar S, Bell ME, Liang J, Soriano L, Nagy TR, Dallman MF. Corticosterone facilitates saccharin intake in adrenalectomized rats: does corticosterone increase stimulus salience? J Neuroendocrinol 2000;12:453-60. 
[24] Bell ME, Bhatnagar S, Liang J, Soriano L, Nagy TR, Dallman MF. Voluntary sucrose ingestion, like corticosterone replacement, prevents the metabolic deficits of adrenalectomy. J Neuroendocrinol 2001;12:461-70.

[25] Miller WL, Tyrrell JB. The adrenal cortex. In: Felig P, , Baxter JD, Frohman LA, editors. Endocrinology and metabolism. 3rd ed.. New York: McGraw-Hill; 1995. p. 555-711.

[26] La Fleur SE, Akana SF, Manalo SL, Dallman MF. Interaction between corticosterone and insulin in obesity: regulation of lard intake and fat stores. Endocrinology 2004;145:2174-85.

[27] Strack AM, Sebastian RJ, Schwartz MW, Dallman MF. Glucocorticoids and insulin: reciprocal signals for energy balance. Am J Physiol 1995;268:R142-9.

[28] Björntorp P. Do stress reactions cause abdominal obesity and comorbidities? Obes Rev 2001;2:73-86.

[29] Hauner H, Schmid P, Pfeiffer EF. Glucocorticoids and insulin promote the differentiation of human adipocyte precursor cells into fat cells. J Clin Endocrinol Metab 1987;64:832-5.

[30] Ottosson M, Lönnroth P, Björntorp P, Edén S. Effects of cortisol and growth hormone on lipolysis in human adipose tissue. J Clin Endocrinol Metab 2000;85:799-803.

[31] Adam TC, Epel ES. Stress, eating and the reward system. Physiol Behav 2007;91:449-58.

[32] Rebuffé-Scrive M, Krotkiewski M, Elfverson J, Björntorp P. Muscle and adipose tissue morphology and metabolism in Cushing's syndrome. J Clin Endocrinol Metab 1988;67:1122-8.

[33] Dallman MF, Pecoraro N, Akana SF, et al. Chronic stress and obesity: a new view of "comfort food". Proc Natl Acad Sci USA 2003;100:11696-701.

[34] Epel E, Jimenez S, Brownell K, Stroud L, Stoney C, Niaura R. Are stress eaters at risk for the metabolic syndrome? Ann NY Acad Sci 2004;1032:208-10.

[35] Pecoraro N, Reyes F, Gomez F, Bhargava A, Dallman MF. Chronic stress promotes palatable feeding, which reduces signs of stress: feedforward and feedback effects of chronic stress. Endocrinology 2004;145:3754-62.

[36] Ulrich-Lai YM, Ostrander MM, Thomas IM, et al. Daily limited access to sweetened drink attenuates hypothalamic-pituitaryadrenocortical axis stress responses. Endocrinology 2007;148: 1823-34.

[37] Foster MT, Warne JP, Ginsberg AB, et al. Palatable foods, stress, and energy stores sculpt corticotropin-releasing factor, adrenocorticotropin, and corticosterone concentrations after restraint. Endocrinology 2009;150:2325-33.

[38] Maniam J, Morris MJ. Palatable cafeteria diet ameliorates anxiety and depression-like symptoms following an adverse early environment. Psychoneuroendocrinology 2010;35:717-28.

[39] Finger BC, Dinan TG, Cryan JF. High-fat diet selectively protects against the effects of chronic social stress in the mouse. Neuroscience 2011;192:351-60.
[40] Prasad A, Prasad C. Short-term consumption of a diet rich in fat decreases anxiety response in adult male rats. Physiol Behav 1996;60:1039-42.

[41] Gibson EL. Emotional influences on food choice: sensory, physiological and psychological pathways. Physiol Behav 2006;89:53-61.

[42] Miller A, Barr RG, Young SN. The cold pressor test in children: methodological aspects and the analgesic effect of intraoral sucrose. Pain 1994;56:175-83.

[43] Leweekowski MD, Ditto B, Roussos M, Young SN. Sweet taste and blood pressure-related analgesia. Pain 2003;106:181-6.

[44] Grigson PS. Like drugs for chocolate: separate rewards modulated by common mechanisms? Physiol Behav 2002;76:389-95.

[45] Kuo LE, Kitlinska JB, Tilan JU, et al. Neuropeptide Y acts directly in the periphery on fat tissue and mediates stress-induced obesity and metabolic syndrome. Nat Med 2007;13:803-11.

[46] de Quervain DJ, Aerni A, Schelling G, Roozendaal B. Glucocorticoids and the regulation of memory in health and disease. Front Neuroendocrinol 2009;30:358-70.

[47] Keller-Wood ME, Dallman MF. Corticosteroid inhibition of ACTH secretion. Endocr Rev 1984;5:1-24.

[48] Houshyar H, Manalo S, Dallman MF. Time-dependent alterations in mRNA expression of brain neuropeptides regulating energy balance and hypothalamo-pituitary-adrenal activity after withdrawal from intermittent morphine treatment. J Neurosci 2004;24:9414-24.

[49] Tomiyama AJ, Dallman MF, Epel ES. Comfort food is comforting to those most stressed: evidence of the chronic stress response network in high stress women. Psychoneuroendocrinology 2011;36:1513-9.

[50] van Strien T, Frijters JE, Bergers G, Defares PB. The Dutch Eating Behavior Questionnaire (DEBQ) for assessment of restrained, emotional, and external eating behavior. Int J Eat Disord 1986;5:295-315.

[51] Kirschbaum C, Pirke KM, Hellhammer DH. The 'Trier Social Stress Test' - a tool for investigating psychobiological stress responses in a laboratory setting. Neuropsychobiology 2008;28: 76-81.

[52] van Strien T, Roelofs K, de Weerth C. Cortisol reactivity and distress-induced emotional eating. Psychoneuroendocrinology 2013;38:677-84.

[53] Tryon MS, DeCant R, Laugero KD. Having your cake and eating it too: A habit of comfort food may link chronic social stress exposure and acute stress-induced cortisol hyporesponsiveness. Physiol Behav 2013;114-115:32-7.

[54] Turner RJ, Wheaton B, Lloyd DA. The epidemiology of social stress. Am Sociol Rev 1995;60:104-25.

[55] Arce M, Michopoulos V, Shepard KN, Ha QC, Wilson ME. Diet choice, cortisol reactivity, and emotional feeding in socially housed rhesus monkeys. Physiol Behav 2010;101:446-55.

[56] Warne JP. Shaping the stress response: interplay of palatable food choices, glucocorticoids, insulin and abdominal obesity. Mol Cell Endocrinol 2009;300(1-2):137-46. 